\title{
Romano, le footballeur gitan aux pieds nus.
}

\section{Quelques traces ethnologiques dans une BD populaire des années 1970 ?}

*Ethnologue, Université d'Aix-Marseille

En France paraît entre 1974 et 1982 pas moins de 31 épisodes de la traduction d'une bande dessinée britannique (Raven on the Wing) sous le titre de Romano ou Romano le Gitan qui racontent les aventures de «Romano le footballeur aux pieds nus ». Chaque épisode comprend entre 46 et 66 planches soit un ensemble d'environ 1700 planches. Romano est une série importante de Trophée, un trimestriel de 164 pages décrit ainsi sur les sites consacrés à la $\mathrm{BD}^{1}$ :

Trophée : 87 numéros de février 1971 à mai 1990.

Revue publiant des séries sportives, principalement axées sur le football. Les deux séries phares étant «Romano » le jeune footballeur gitan jouant pieds nus de Tom Tully (scénario) et Francisco Solano Lopez (dessins) et « Hamish la foudre ", Le footballeur écossais aux shoots meurtriers Hot Shot Hamish dans la version originale. Deux bandes d'origines britanniques.

Trophée est une revue de bandes dessinées petit format parue chez l'éditeur Aventures \& Voyages. Cette maison d'édition fondée en 1946 est spécialisée dans la publication de revues de bandes dessinées destinées aux enfants et adolescents, parmi les dizaines de titres certains lecteurs se souviendront peut-être de Akim, Lancelot, Marco Polo, Ivanboé, En piste, etc. Il s'agit donc de bandes dessinées extrêmement populaires mais qui n'ont pas la légitimité des « grandes » revues de l'époque comme Pilote, Tintin ou Spirou. Les auteurs de Romano sont des créateurs reconnus, Tom Tully (scénario) et Francisco Solano Lopez (dessins). Ce dernier (1928-2011) publiera aussi chez Dargaud avec Carlos Sampayo comme scénariste. On peut situer leur production dans la grande tradition des feuilletons populaires à rebondissements multiples et dont souvent l'épisode de termine par l'invitation à attendre, avec impatience, le numéro suivant pour savoir comment le héros va se sortir d'une situation aussi inextricable que dramatique. La liberté dont jouissent les auteurs est lisible dans le scénario comme dans le dessin. Pour tenir le lecteur en haleine le scénariste peut mêler le réalisme social et le fantastique, le dessinateur adopte une mise en forme très cinématographique, pouvant alterner des gros plans, des plans larges et des contre-plongées, jouant du noir et blanc, les ombres sont tranchées, signifiantes. Les personnages sont très «typés ", parfois stéréotypés mais pouvant aussi sortir de cela pour en être plus 


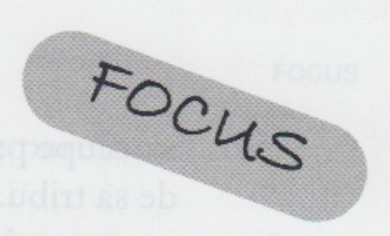

surprenants voire attachants. On est loin de la «ligne claire»; lire aujourd'hui ces $\mathrm{BD}$ permet de se rendre compte que ces productions à destination des adolescents mâles des années 1970-1980 esquissent un mouvement, dans la $\mathrm{BD}$, identique à celui que connaît au même moment le roman policier qui devient " polar » puis « roman noir » et une part du jazz, avec le free ou la «pop-music» violentée par le punk et le disco.

\section{Romano}

Le personnage de Romano apparaît dans le n 15 daté d'août 1974 jusqu'au n 46 de mai 1982. L'accès à l'ensemble des épisodes ne paraît guère facile à moins d'aller à la $\mathrm{BnF}$. Il a été possible d'en lire six (un exemplaire papier provenant du fonds Joseph Valet grâce auquel l'idée de cet article a émergé, et cinq récupérés sur un site de partage d'internet).

« Romano est un gitan de la tribu des Lengros, possédant une usine de jouet dont la principale occupation est le football. Un sport où il excelle bien qu'il joue pieds nus. On peut donc le suivre à travers les péripéties de son équipe de Highboro. Romano est également très superstitieux et Morag, la sorcière de sa tribu a une grosse influence sur lui. Il est aussi extrêmement misogyne, voire macho. » Ainsi est présenté le personnage sur les sites web ${ }^{2}$ consacrés à la bande dessinée.

S'adressant à un public de jeunes garçons il n'y a effectivement peu de place pour des personnages féminins hormis la fille du directeur du club, jolie potiche blonde qui mène la troupe de pom-pom girls et la grandmère de Romano, "Morag, la sorcière de la tribu des Lorengros » qui apparaît aux moments décisifs pour sauver Romano, toujours couverte d'un drap noir qui masque son visage et un corbeau sur l'épaule ou virevoltant autour d'elle; l'oiseau noir est l'esprit du clan, le corps de Romano porte une marque de naissance le représentant, il peut " apparaître » à Romano dans les moments critiques comme un signe pour se diriger géographiquement ou comme la voie (la voix) à suivre pour respecter la « loi de la tribu». On aperçoit là un des éléments importants du ressort dramatique de la série, le fantastique projeté sur la réalité gypsy. Celle-ci est largement présente par divers aspects bien réelle de la vie des Travellers, bien entendu mêlée à l'inévitable série de fantasmes associés, là comme ailleurs, à ce monde. Le troisième ressort est l'origine (cachée) et le devenir (incertain, semé d'embûches) de Romano dont on apprend qu'il est " propriétaire d'une usine de jouets » ce dont il ne 
s'occupe pas n'ayant que deux intérêts : le foot et le respect des traditions de sa tribu. Chaque nouveau jouet sortant de "son » usine, le passionne, comme sûrement les lecteurs de la revue qui ont le même âge que le héros, tellement qu'il parait bien infantile par l'attention qu'il y porte au regard des enjeux dont il est le centre. Il ne se rend pas compte que c'est dans l'entreprise et le club de foot même que se trouve son plus grand ennemi qui veut le déposséder de son bien. Et le nécessaire ressort, indispensable pour chaque épisode, est le déroulement du match de foot du jour, avec toutes les péripéties invraisemblables possibles et les prouesses exceptionnelles du « footballeur aux pieds nus ».

Le personnage de Romano apparait pour la première fois en couverture du n 15 de Trophée. Il est représenté en tenue de footballeur, il n'est pas encore le joueur aux pieds nus mais le décor indique très clairement qu'il est " gitan ", l'arrière fond est un champ dans lequel sont installées trois roulottes (couverture Trophée 15).

Quand le personnage est bien installé dans la série il apparaît pour ce qu'il est vraiment, le footballeur aux pieds nus (image couverture Trophée 28).
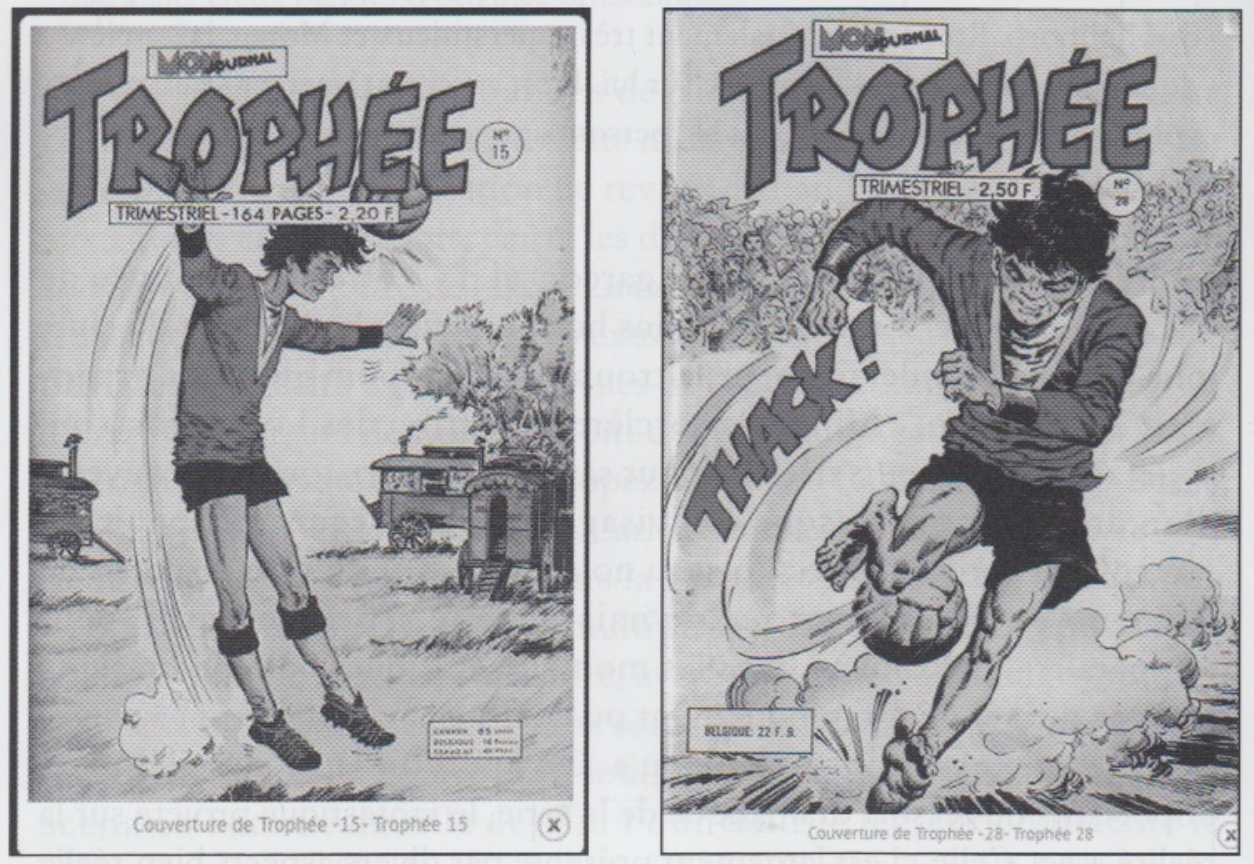

\section{Vocabulaire}

Cette bande dessinée est traduite de l'anglais, sans indication du ou des traducteurs. On ne sait donc pas quels termes désignent Romano et les membres de sa tribu dans la version originale. Les mots utilisés 
en français sont bien ceux du français courant des années de parution de la série :

- "gitans ", dialogue en l'entraîneur et le président du club :

L'entraîneur : « Un message de Romano! Il dit que sa tribu doit se rendre à une fête gitane à trente kilomètres... »

Le président : Depuis quand ce maudit vagabond prend-il des décisions sans nous consulter! C'est l'anarchie!

L'entraineur : les gitans n'acceptent aucune contrainte ... » (n³4, p. 21)

- «bohémiens ${ }^{3}$ », un footballeur s'adresse à Romano : «Nous faire battre par une bande de bohémiens, je voudrais bien voir ça!» $\left(\mathrm{n}^{\circ} 37\right.$, p. 47$)$; l'entraîneur aussi qualifie ainsi Romano : «Ce sacré petit bohémien me donne des sueurs froides chaque fois qu'il fait ce genre de truc !» ( $n^{\circ} 38$, p. 16).

- «manouches » - un joueur s'exclame : «Des manouches, rien que des manouches, on finira par jouer sur des terrains vagues! " à quoi le responsable du club répond : «Ouais!... Romano est un manouche comme tu dis ! N'empêche qu'il est le meilleur joueur du championnat!» ( $\mathrm{n}^{\circ} 38$, p. 47). Parfois Romano se fait injurier : "sale manouche » ( $n^{\circ} 37$, p. 45$)$.

- «rom» - au cours d'un match un jour s'exclame : "Gaffe les Loulous : le rom est en train de nous souffler la balle » (n³8, p 57). Le terme rom ne sert qu'à qualifier Romano, il n'est pas employé au pluriel pour désigner la tribu ou d'autres personnages.

Si les termes bohémien, manouche et gitan sont bien synonymes dans le français courant des années 1970-1980, il est plus étonnant que le mot rom apparaisse car il n'était pas courant à l'époque, et plutôt employé par des gens ayant affaire au monde du voyage et sachant distinguer les «tribus ». Le fait qu'il ne soit utilisé que pour qualifier Romano vient peut-être de l'original anglais.

Quelques termes de la "langue des gitans » apparaissent au fil des épisodes consultés, il y en aurait sûrement d'autres en épluchant tous les numéros de la série. Le mot gadjo, gadjos au pluriel, est utilisé par Romano: «Félicitations, boss! Vous êtes peut-être un vulgaire gadjo ... mais vous êtes un sacré entraineur » (n³8, p.32); "Que le hérisson sacré te ronge les oreilles, gadjo! ... Je ne serai jamais le larbin des gadjos! Porte tes affaires toi-même » $\left(n^{\circ} 37, p .8\right)$. "Gadjo » n'est pas courant en français dans les années 1970-1980, il ne figure pas encore dans les dictionnaires (Petit Robert, édition 1983) alors que c'est le cas aujourd'hui ${ }^{4}$. « Vardos » désigne les roulottes (verdine, verdon). Dans des exclamations apparaissent des mots « romani ». Un footballeur gitan va disputer un match contre l'équipe de Romano, « Il s'appelle Ignatus Zanco ${ }^{5}$. Il 
fait partie de la tribu des Vengali ... les gitans les plus primitifs d'Europe !» ( $\mathrm{n}^{\circ} 18$, p. 39). Il jure : «Par la barbe du Drabhi! Le signe du phurdini! la patte de belette ! ... C'est le signe maudit du démon souffleur !». Romano n'oublie pas qu'il est « gitan » : «Ça va barder par Sara !» (n³6, p. 21).

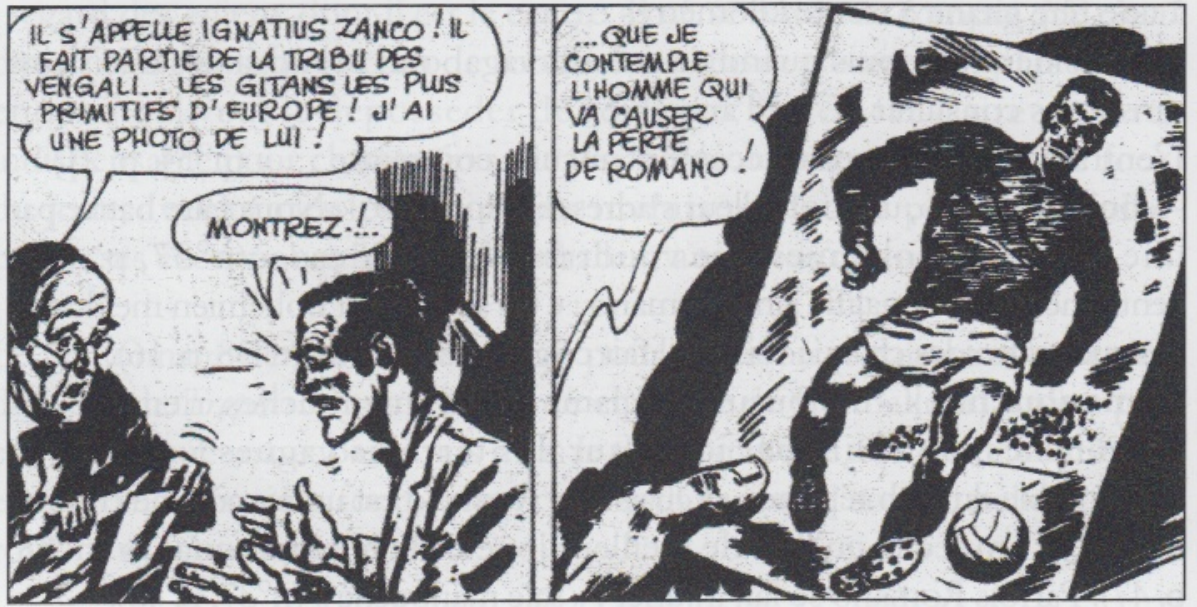

Il ne fait aucun doute que le nom de Zanco n'est pas dû au hasard. Parmi les Roms qui passent en Angleterre en 1914 (sous les noms de Koudakoff, Sotnikoff, Kalmikoff et Filipoff) un Zanco est photographié à Whitechapel ${ }^{6}$ (voir annexe).

\section{La culture «tsigane »}

Par nombre de faits émaillant le récit des exploits de Romano on s'aperçoit que le scénariste a une certaine connaissance du monde «tsigane» mais que bien entendu il profite des caractéristiques connues ou supposées de ce monde pour mêler le vrai, le vraisemblable et le fantaisiste voire le fantastique.

Les aventures de Romano se déroulent dans un temps indéterminé ; aucune date, aucun objet (modèle de voiture par exemple) ne permet d'identifier autrement que "maintenant» la période. L'univers de vie de la tribu de Romano rappelle le «temps des chevaux » selon l'expression des Manouches d'Auvergne. Elle vit en "vardos ", avec des chevaux et des ânes, elle a des problèmes pour trouver où stationner et grâce à Romano pourra être accueilli dans le parc de M. Mortimer, le propriétaire du club de foot.

\section{Les lieux}

\section{Les espaces gadjos}

Le déroulement des aventures de Romano se passent pour une très bonne part sur le terrain de football et dans les vestiaires. Le terrain de foot, 


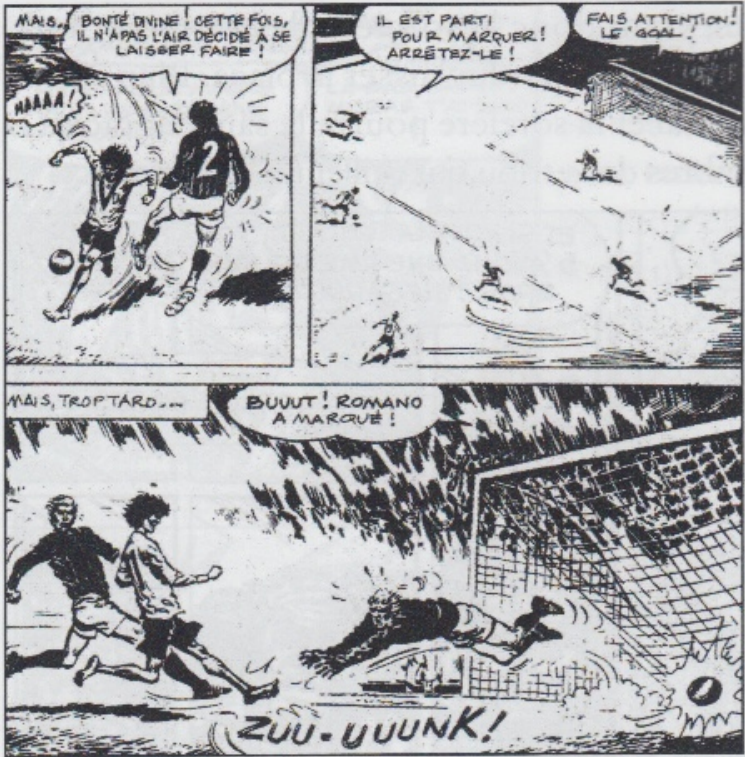

pour l'entrainement mais surtout pour les matches qui ont toujours un enjeu décisif pour l'équipe qu'elle soit celle des Lengros, des gitans quand ils affrontent les autres équipes de jeunes de la ville ou de la région, celle du club professionnel dont Romano est la vedette ou bien même de l'équipe d'Angleterre quand Romano est sélectionné.

La vie de Romano est liée à celle de sa tribu, c'est une vie de gitans. Il y a de l'entre-soi, le campement sera plusieurs fois représenté, de loin ; il est situé à la périphérie de la ville voire en pleine campagne. Pour autant, Romano ne cesse d'avoir des contacts avec des gadjos, de circuler dans l'espace de ceux-ci, la ville.

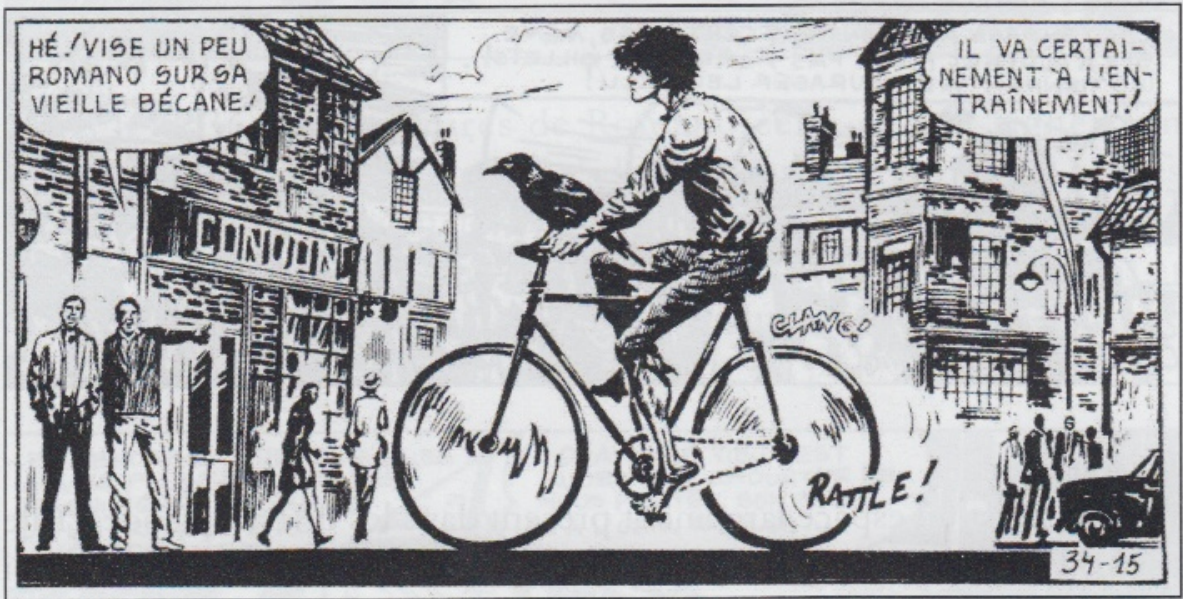

D’autres espaces « gadjos » dans lesquels Romano va passer sont dessinés. C'est le cas de l'usine de fabrication de jouets dont il a hérité (mais dans les épisodes consultés, $20 \%$ de l'ensemble, il n'y a pas moyen de comprendre de quoi il s'agit et aucune image la représentant)

Romano va être victime de très graves brûlures en sauvant des chevaux (Trophée $\mathrm{n}^{\circ} 18$, «L'ombre de Zanco »). Il est amené à l'hôpital où les 
médecins sont impuissants, vient donc la sorcière de la tribu pour le soigner mais les médecins ne veulent pas lui laisser la place. Bien sûr un gitan n'est jamais seul à l'hôpital et la sorcière pourra le sauver grâce à la pression silencieuse des membres de la tribu qui veillent à l'extérieur.

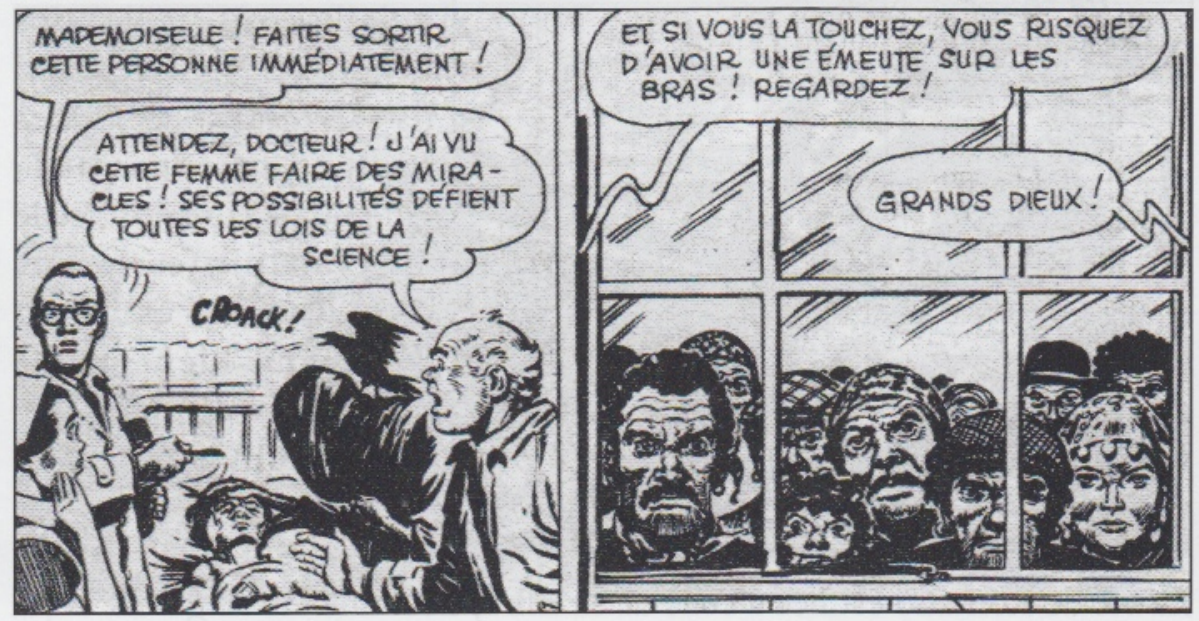

Comme dans tous les moments importants, il n'est pas question de laisser seul Romano aux milieux des gadjos. Ainsi quand Romano est sélectionné pour jouer dans l'équipe d'Angleterre, ce sont les Lengros qui arrivent aux abords du stade et pas simplement sa famille.

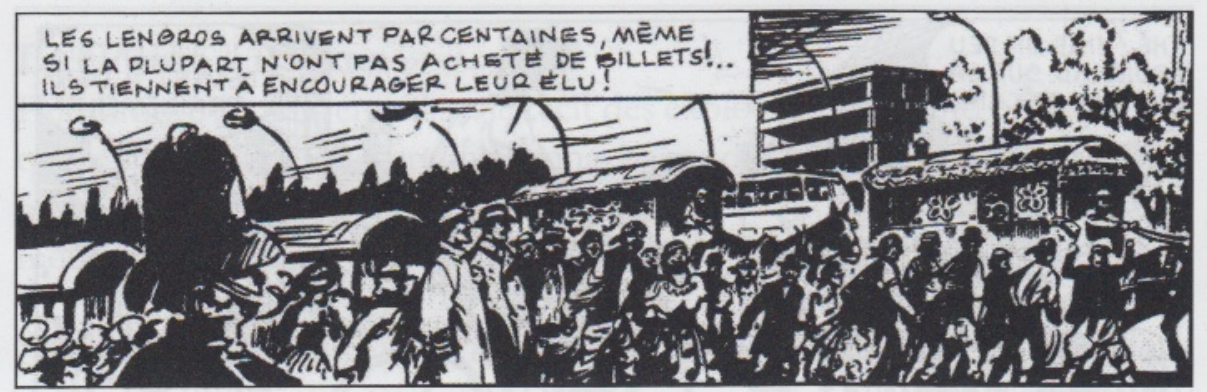

\section{La nature}

La nature est un espace largement présent dans les divers épisodes. Elle est le lieu de vie de la tribu, pour l'installation du campement mais aussi un site précis, ressemblant étonnamment à Stonehenge, est indiqué comme celui des pèlerinages de printemps de tous les gitans du pays. La nature permet l'apparition du " merveilleux », des signes qui vont interférer sur la vie de Romano. Les gitans de la bande dessinée vivent comme tous les «Gitans » du monde au milieu des gadjé, pourtant certains espaces deviennent romanès par l'occupation temporaire, de longue durée (campement) ou occasionnelle (l'hôpital). 


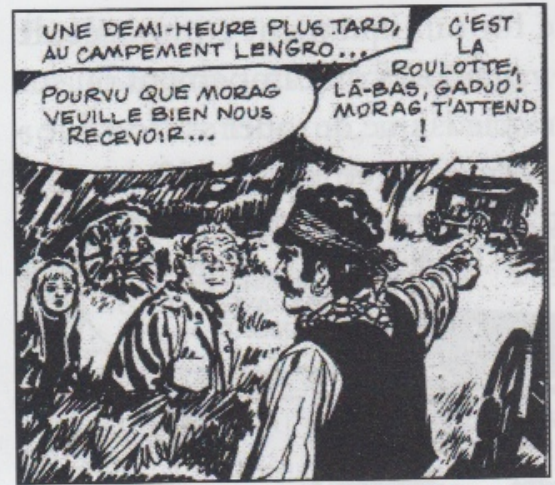

Dans plusieurs épisodes on a l'occasion d'apercevoir le «Campement lengro », soit parce que Romano y est ramené auprès de sa grand-mère pour qu'elle le soigne ou le libère des sorts qui lui ont été jetés, soit encore quand l'entraineur du club vienne à sa recherche.

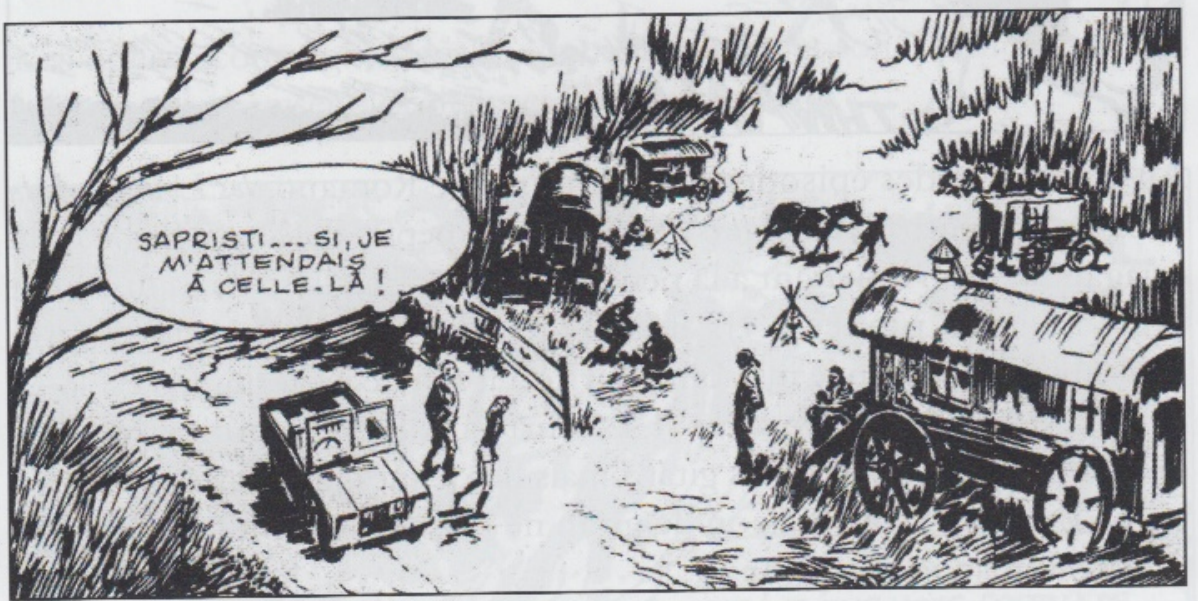

Dans le $n^{\circ} 36$ des aventures de Romano celui-ci doit signer son réengagement dans le club mais il s'adresse à l'entraîneur du club par ces mots : «... M'sieur Hagan, je voudrais que vous accordiez une faveur à ma tribu ... les Lengros vont bientôt célébrer la fête du printemps et maintenant qu'on nous a chassés de notre campement pour y construire des HLM, il nous faut un nouveau terrain » ( $\left.n^{\circ} 36, p .14\right)$ :

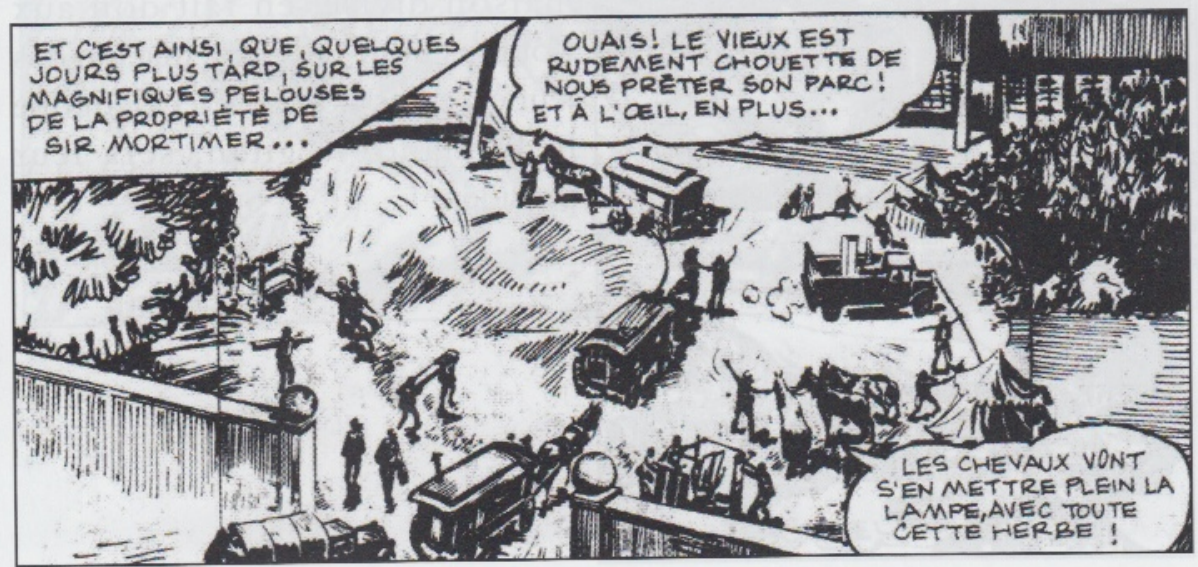


Quand Romano entraîne une équipe de foot uniquement composée de jeunes de sa tribu il le fait sur un terrain vague près du campement ou sur une plage : «Il n'y a rien de mieux pour les pieds que de jouer sur le sable » $\left(n^{\circ} 36, p .24\right)$ ou bien de s'entrainer sur un terrain vague $\left(n^{\circ} 37, p .52\right)$ :

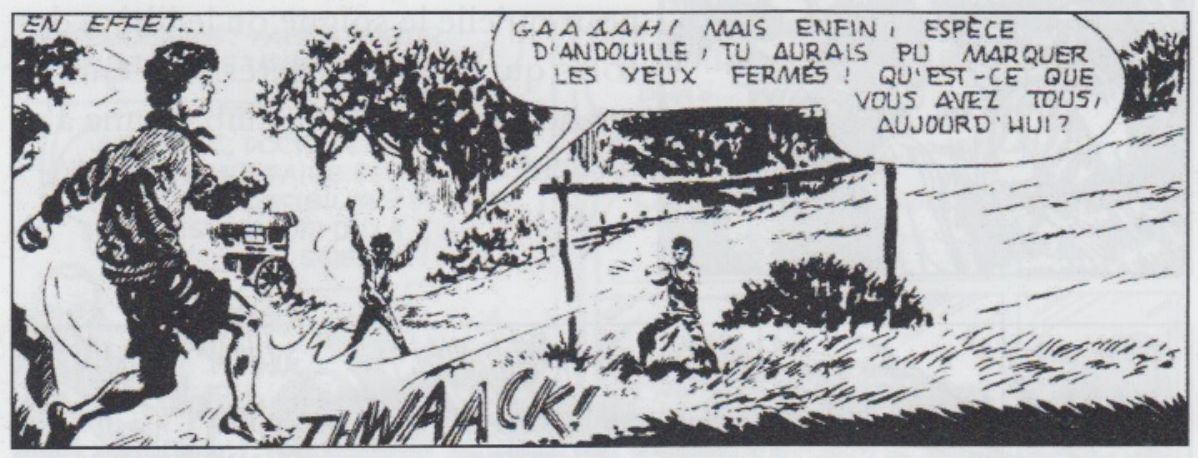

Au cours d'un des épisodes des aventures de Romano, les Loulous de la décharge, un footballeur adverse signifie son mépris du "manouche », du «vagabond » en l'invitant à la pendaison de crémaillère de sa luxueuse villa. Le gitan piqué au vif décide de relever le défi et de se faire construire lui aussi une maison, pour cela il achète un terrain, c'est la ... décharge de la ville! Du coup il se heurte aux usagers ordinaires de la décharge qui ne sont pas les gitans mais des jeunes prolétaires irlandais, les «Loulous». Une maison de gitan ne peut ressembler à celles des gadjos, Romano a son plan :

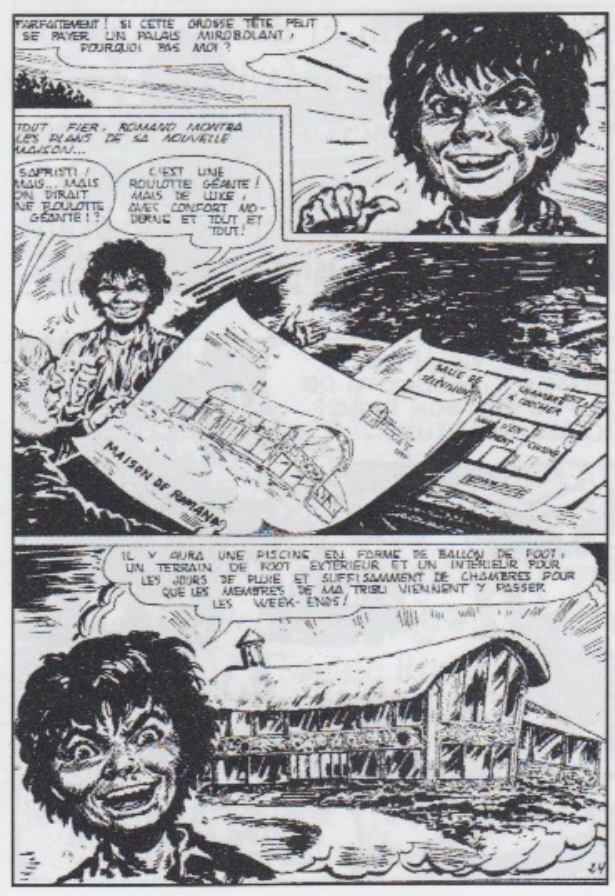

La maison-roulotte construite sur la décharge devient une pierre de discorde avec les « Loulous». Cela doit se régler par un match de foot que gagnent les gitans. Romano, magnanime, et constatant que la maison divise, en fait don aux Loulous pour faire une maison des jeunes, et les Loulous décident que le Romano, le gitan, sera leur président! 


\section{Bestiaire}

Les animaux sont parfois présents dans les aventures de Romano. Ils ne sont pas nombreux, mais comme tous les épisodes n'ont pu être lus sûrement en manque-t-il. Il n'y a ni chien, ni chat mais une corneille, des chevaux et des ânes, un bout de belette et l'évocation plusieurs fois répétée du hérisson.

Les chevaux sont attelés aux verdines, et au début d'un épisode particulièrement dramatique Romano sauve, au péril de sa vie, plusieurs chevaux d'un ranch voisin.

Les ânes accompagnent les chevaux mais surtout sont les montures des courses qu'organisent les gitans et leurs voisins.

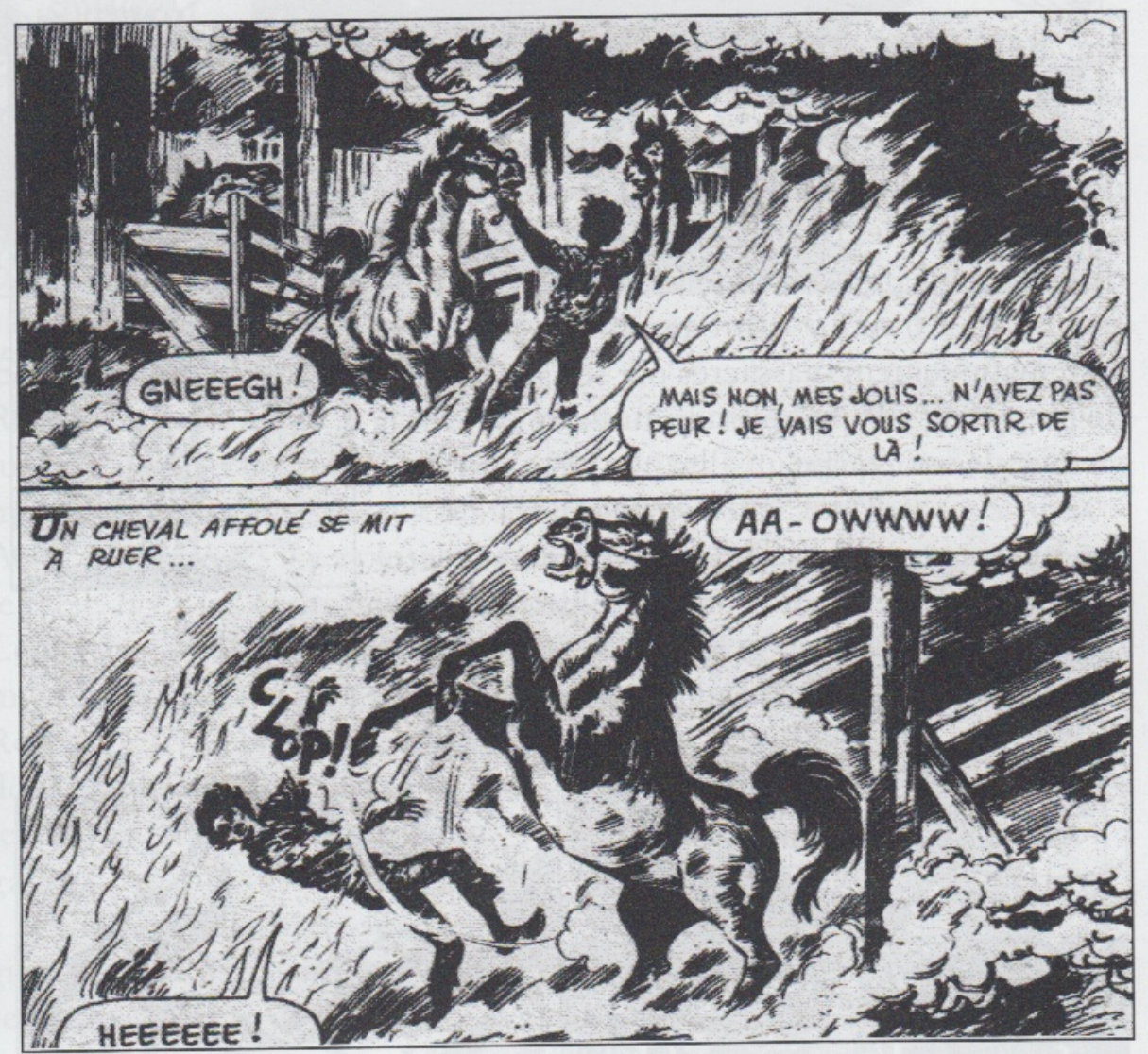




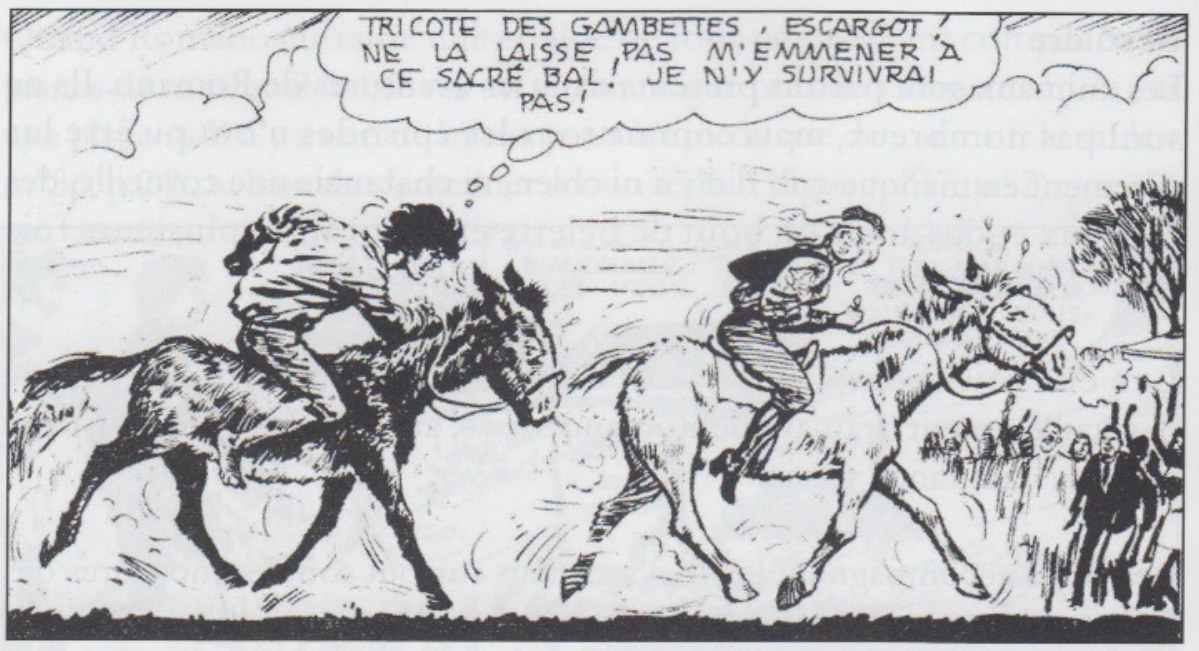

Le hérisson est plusieurs fois là, mais uniquement sous forme symbolique. Il n'est pas une nourriture (on ne mange pas dans le monde de Romano). Il est invoqué : "Que le hérisson sacré te mange les oreilles, gadjo » (n³7, p. 8). Il a des vertus magiques, du moins ses excréments et permet à Romano dont les pieds nus sont systématiquement massacrés par ses adversaires, de récupérer en quelques minutes et assurer la victoire de son équipe.

La belette, sous la seule forme de sa patte séchée, est le véhicule d'une malédiction qui va

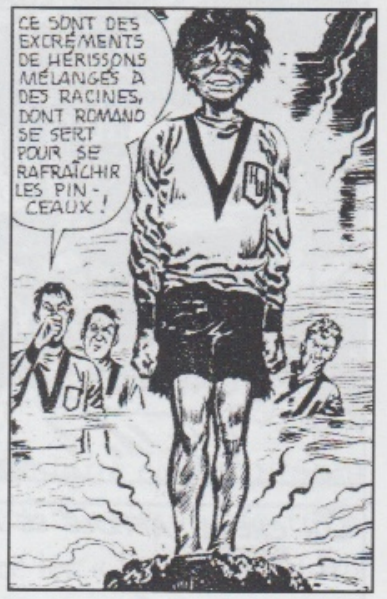
déclencher la guerre entre Romano et Zanco (gitan espagnol d'une équipe rivale) : «C'est le signe maudit du démon souffleur ! »

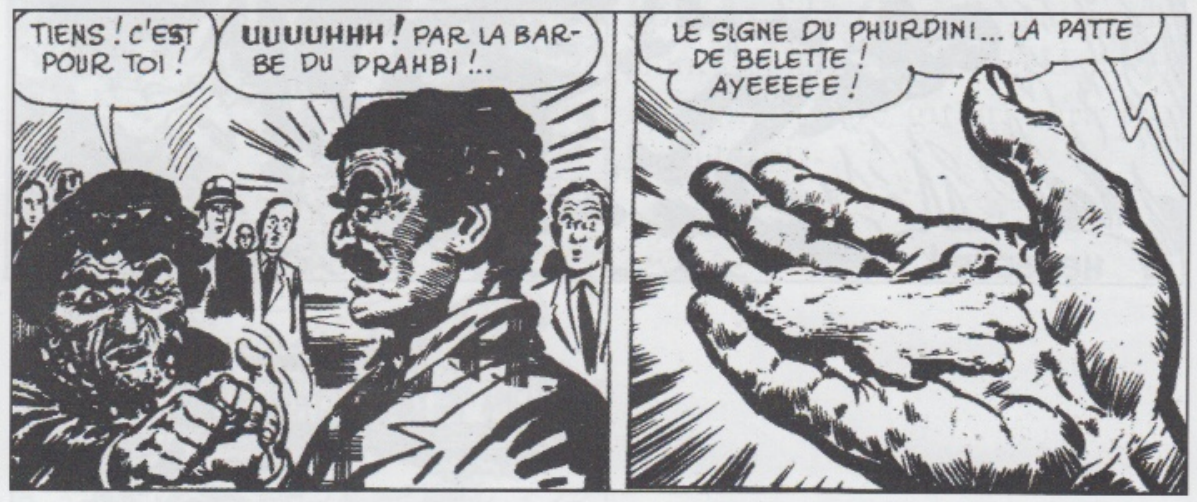


Et enfin la corneille. Celle-ci est le symbole de l'esprit de la tribu, elle accompagne toujours la sorcière qui veille sur le respect des traditions et aussi sur Romano. Elle se manifeste à Romano pour lui indiquer le bon chemin - symbolique, elle lui fait se remémorer les «lois », réel il lui arrive d'apparaître pour le guider dans la bonne direction. Elle est aussi le messager de Romano pouvant transmettre aux bonnes personnes un message qu'il tient dans son bec. Romano est « l'élu » de la tribu, il porte une tache de naissance qui a la forme de la corneille, " totem » de la tribu.

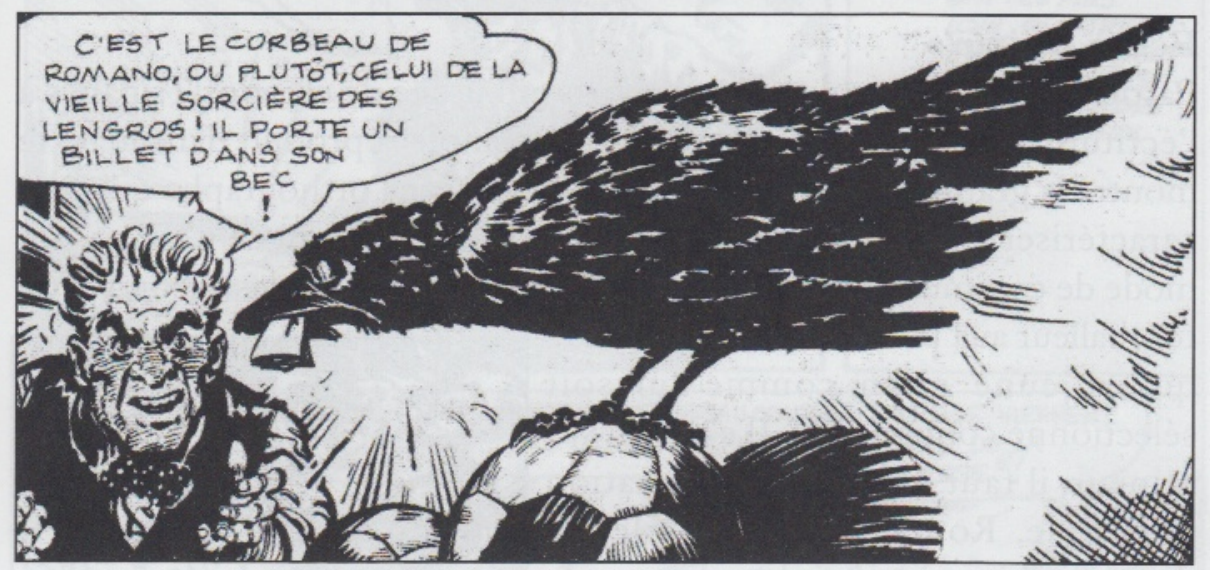

\section{Écritures}

Romano ne sait ni lire ni écrire, ou presque ... mais il parle " gitan ", cela lui sert sur le terrain pour donner ses conseils quand il joue avec des membres de sa tribu, sans que les adversaires puissent anticiper ses astuces. Au fil des épisodes il y a plusieurs fois des écrits qui circulent et pimentent le récit. Il y a les écrits des gadjos sur les gitans, en particulier sur "Les Lengros, une peuplade mystérieuse", la tribu de Romano. Il y a la manière dont les gitans écrivent des lettres dans la langue des gadjos; et puis il y a les "écritures " propres des gitans, ce qu'ils savent eux seuls lire.

\section{Les livres savants}

Pour percer les secrets des gitans, les gadjos peuvent avoir recours à des livres

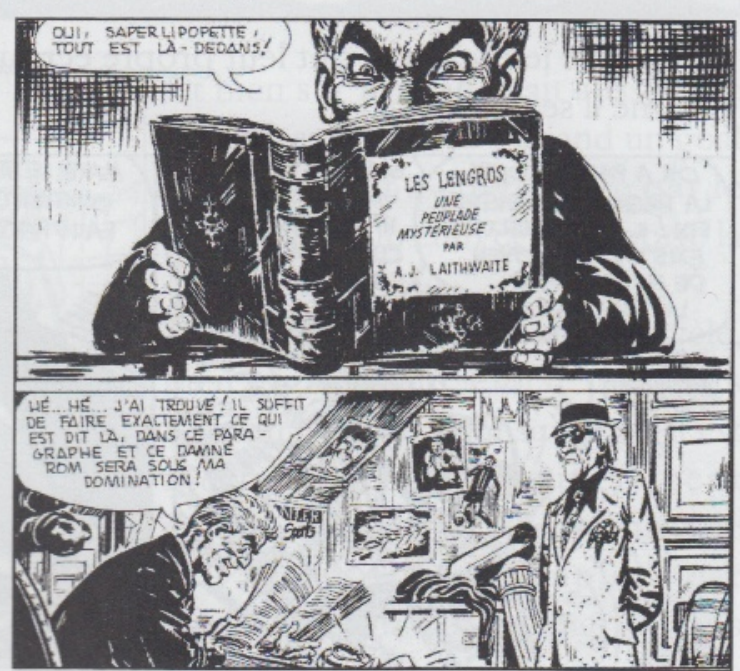


qui permettraient de percer le secret de leur langage codé par exemple, mais cela ne fonctionne pas toujours. Pourtant dans un épisode, un gadjo va mettre la main sur le livre des Lengros, une tribu mystérieuse. Un ennemi de Romano va savoir y puiser des informations afin de le contraindre à respecter les «lois » et ainsi tomber sous son pouvoir

Sans que cela soit dit explicitement, mais le lecteur le comprend bien, les livres « d'ethnologie» ou de folklore peuvent être un danger pour les « gitans ».

\section{Écrire "gitan "}

Patrick Williams a analysé les rapports et l'usage de certains Tsiganes à l'écriture dans un article de $1997^{7}$. Un exemple reproduit dans le texte montre la graphie particulière, la distance d'avec l'orthographe qui peut caractériser les productions scripturaires de ceux dont ce n'est pas un mode de communication habituel. Dans l'épisode $n^{\circ} 38$ des aventures du footballeur aux pieds nus, Romano veux qu'un jeune gitan comme lui soit sélectionné comme goal. Il est bien sûr mineur, il faut donc une autorisation parentale. Romano exhibe la lettre d'autorisation du père du garçon et le dessinateur la reproduit en assez gros pour que les jeunes lecteurs de la revue puisse la lire et comprendre par sa graphie et son orthographe qu'elle est authentique ; elle est écrite en français mais à la manière « gitan» :

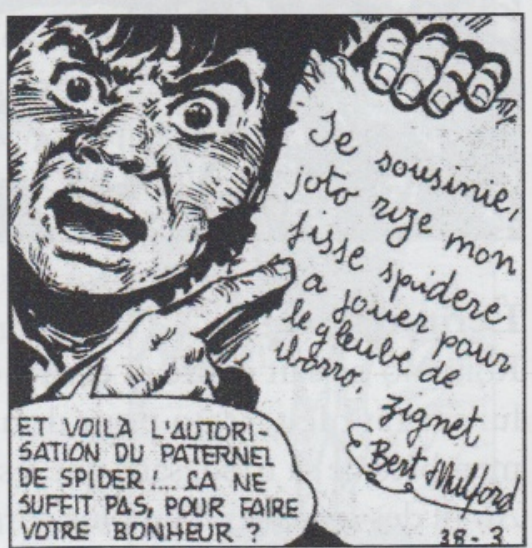

Par contre les Lengros ont leur propre écriture, secrète et indéchiffrable comme il se doit :

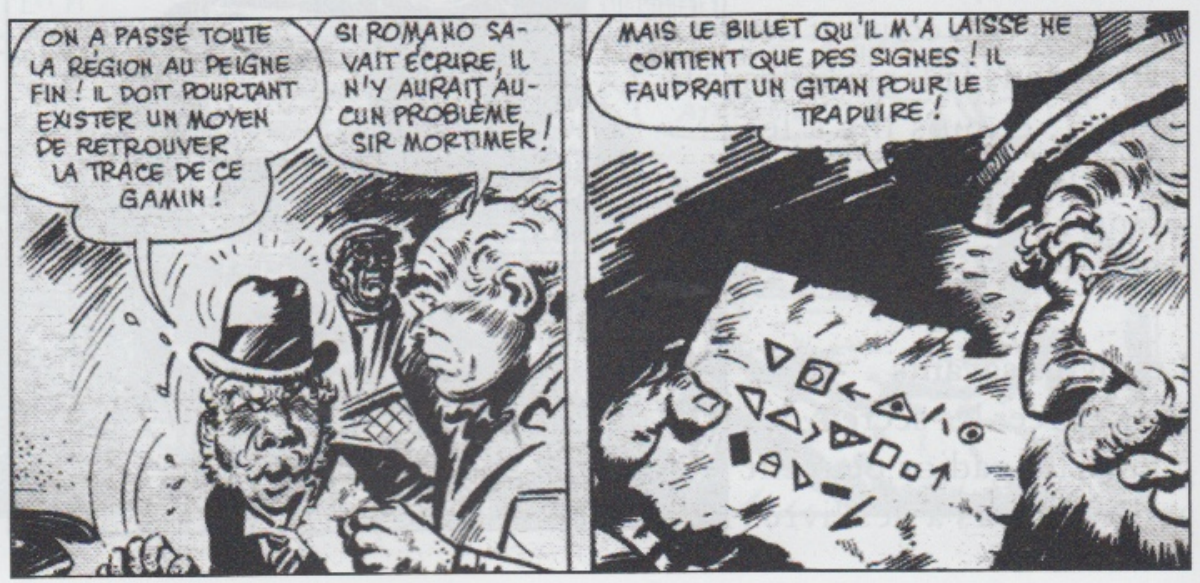


Le « savoir lire gitan"

Il est bien connu que les gitans, étant nomades, doivent laisser des signes sur les bords des routes pour pouvoir se retrouver. Et seul un membre de la tribu comprend ce que peuvent signifier des cailloux apparemment éparpillés sur le sol:

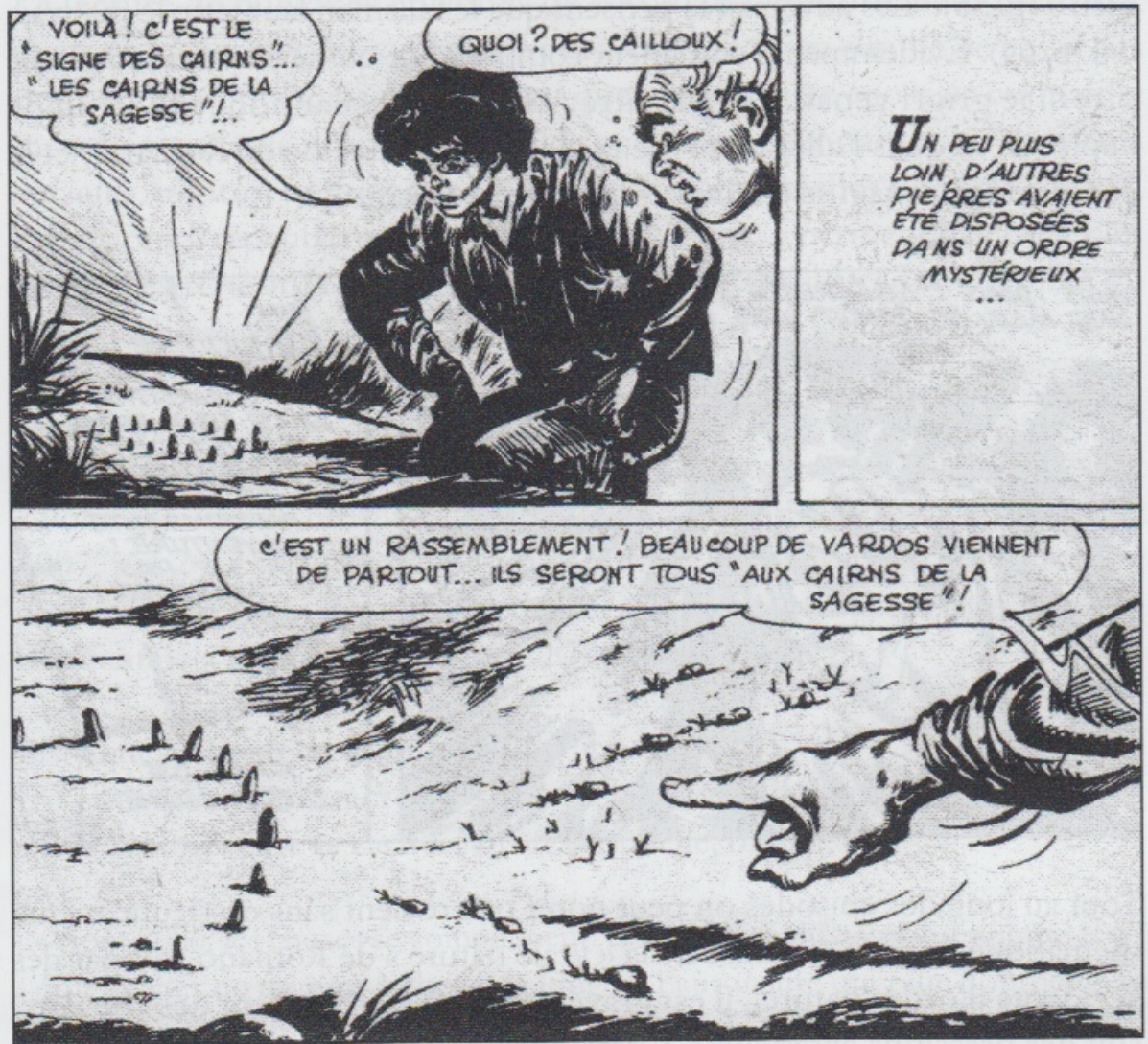

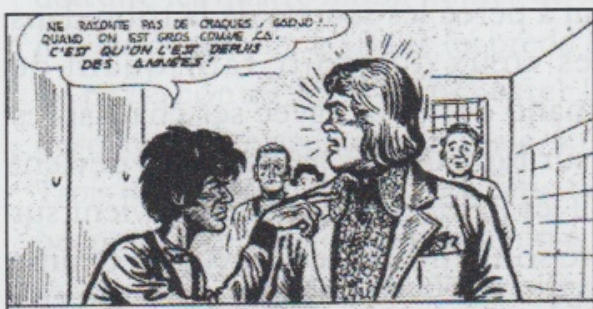

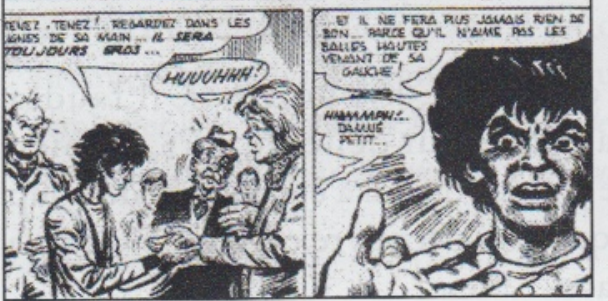

Et bien sûr Romano sait aussi « lire les lignes de la main » quand un goal incompétent a été recruté par le propriétaire du club et en apporte la "preuve » :

La prédiction de Romano se révèle, bien évidemment, exacte. Au cours du match de foot qui suit le goal est inefficace, et particulièrement vulnérable sur les ballons venant de sa gauche. Il sera remplacé par Spider, de la tribu des Lengros. Mais il est inexpérimenté ... et très superstitieux. 


\section{Superstition}

L'épisode s'intitule « Le talisman ». Spider, goal manouche tout gringalet est embauché, il est terrorisé. En plein milieu du match, Romano va chercher le porte bonheur du gamin et explique à un autre joueur qu'il s'agit d'« un talisman qui ne le quitte pas depuis sa naissance ... sans lui, il ne se sent pas bien » (p. 55). Les adversaires pensent que le talisman leur jette le mauvais œil (p. 64). Évidemment l'entraîneur comprend ce qui se passe et redoute le pire si le grigri venait à disparaître : chose qui est annoncée à la fin de l'épisode : "dans l'allégresse (de la victoire de l'équipe de Romano) et la confusion une main se tendit vers le précieux talisman et ...»

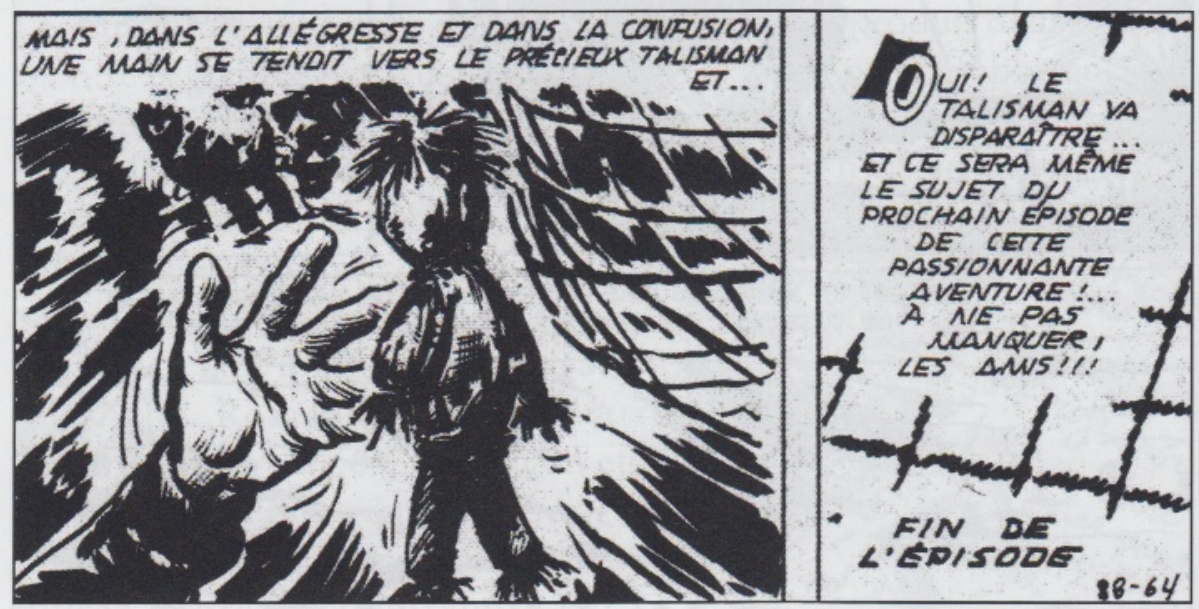

Tout au long des épisodes on peut noter que revient sans cesse une même thématique, la mise en cause de la «vraie nature » de Romano soit par des accidents d'ordre naturel, il est gravement brûlé en sauvant des chevaux, soit des pressions extérieures, il est obligé de respecter la loi des Lengros et de réaliser trois vœux d'un gadjo qui a percé les secrets de la tribu dans un livre (voir plus haut). Parmi ces vœux du gadjo, fabriquant de chaussures de sport, il est dit que Romano doit jouer avec ses chaussures ("Chantage publicitaire » $n^{\circ} 32$ ). Bien sûr Romano n’y arrive pas, il devient mauvais et ce n'est qu'au moment où il les jette dans la foule et revient sur le terrain pieds nus qu'il retrouve toute son efficacité et sauve son équipe à le dernière minute.

D'autres fois il tombera sur un charlatan, hypnotiseur, il faudra l'intervention de la sorcière de la tribu pour délier le sort, ou bien quelqu'un vole la "pierre sacrée » de la tribu qui lui a été confiée du coup il est banni par les siens jusqu'à ce que l'on découvre qu'il a été victime d'une manipulation et retrouve sa place. 
La supertition paraît bien un univers partagé entre les gitans et les gadjos. Les pouvoirs que les uns accordent aux autres, dans les deux sens, fondent leurs rapports tout à la fois de confrontation, de défiance et parfois de coopération.

La lecture de quelques épisodes de « Romano le footballeur gitan aux pieds nus " permet de comprendre la structure assez simple de ces récits pour adolescents mais révèle aussi que le scénariste écossais connaît le monde gitan, ses rapports avec la société environnante, les tensions sociales, injures, préjugés, mais aussi difficultés réelles du mode de vie, expulsions à répétitions, etc. Cette trame " gitane " permet de dérouler les récits très répétitifs des matchs de football dans un cadre qui mêle du « sociologique » au réalisme, du fantasmatique et du fantastique.

\section{Épilogue}

La revue SO FOOT au cours de l'été 2007 consacre un dossier au sujet «Foot et Gitans » intitulé « Marquage à la roulotte » ( $n^{\circ} 46$ - juillet-août 2007, pp. 24-31). Il n'y est pas question de Romano mais l'on retrouve plusieurs anecdotes et jugement de valeur sur les footballeurs gitans qui se trouvent déjà dans les épisodes de Romano.

Juste quelques exemples.

Un encadré est consacré (p. 26) à " Eastwood, le Gitan qui tire juste », après avoir résumé sa carrière professionnelle, on lit:

" Août 2006, les choses se compliquent. La notoriété d'Eastwood grimpe et il doit arrêter de monter à cheval le long de l'autoroute qui relie Southend à Londres. Et puis cette décision de la mairie de Basildon qui menace de détruire les quatre mobil homes de sa petite communauté pour occupation illégale du terrain. L'affaire lui prend la tête. Son rendement baisse. Son coup de patte contre MU (Manchester United) lui sauve la mise. Freddy est désormais une vedette nationale. Et le conseil municipal se sent merdeux. Trois jours après l'exploit, la mairie fait savoir à Freddy qu'il peut continuer à occuper son terrain pendant cinq ans. Mais la municipalité la joue fine en assortissant l'autorisation d'une obligation pour les occupants de rester unis. Si l'un deux quitte le terrain, l'autorisation prendra fin. Les clubs du Premiership qui ont lorgné sur le nouvel international gallois vont devoir remballer leur chéquier. «Il est très rapide et difficile à marquer, dit de lui Steve Tilson, son entraineur. À moins de l'attacher à sa roulotte ». 
Des joueurs gitans, Antonio et Ivan Amaya, font part au journaliste de leur opinion :

"Moi, je dis que nous avons le même style de jeu que les Brésiliens. On joue dans les rues comme eux, on court pieds nus, on fait des cages avec des bouteilles, et on est aussi technique qu'eux. La seule différence entre eux et nous, c'est que nous avons plus de conilles». Ce qu'Tvan, qui lui est passé pro, (une plus grosse paire?) traduit par: "On a tendance à se laisser la peau sur le terrain. On a envie de prowver aux gens qu'on est bon » (p. 29).

Et Jesus Seba, ancien joueur du Real Saragosse, de Vilarreal et de Wigan, observe :

"...on peut dire qu'à valeur égale, les clubs choisiront toujours celui qui n'est pas gitan.. Pour eux, c'est plus sûr, car ils voient le Gitan comme quelqu'un d'imprévisible. S'il l'est dans la vie, pourquoi il ne le serait pas sur le terrain?"

Romano, le gitan imprévisible, footballeur aux pieds nus n'est pas loin !

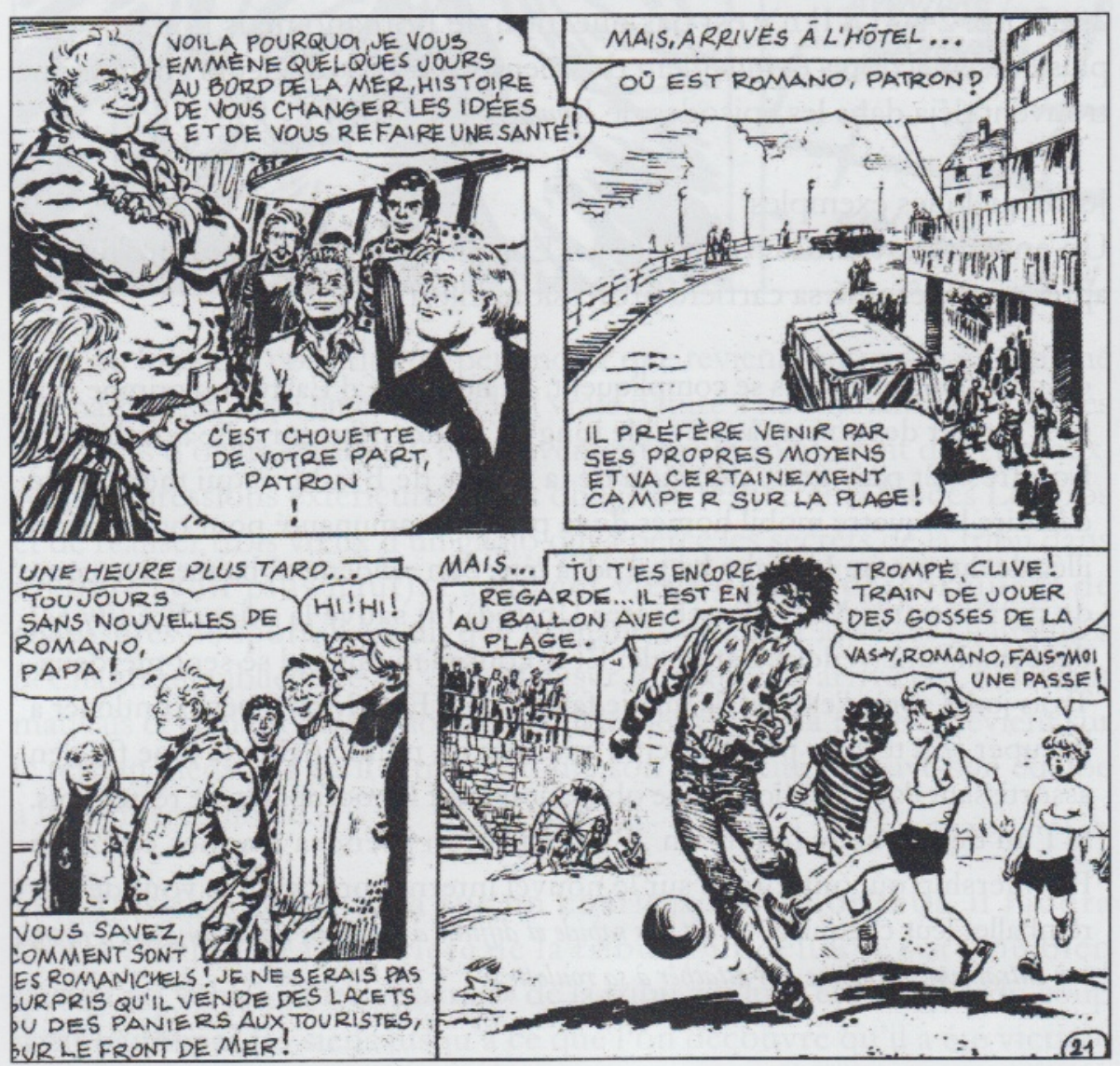

Suite au prochain épisode $\ldots{ }^{8}$ 
Listes des titres des épisodes (et parfois résumés) des aventures de Romano, le footballeur gitan aux pieds nus parus dans le journal Trophée entre août 1974 et mai 1982, (en gras les épisodes consultés).

$\mathrm{N}^{\circ} 15$ le scandale de Highboro

$\mathrm{N}^{\circ} 16$ Le signe des Kaldashs

$N^{\circ} 17$ Mauvaise rencontre

$N^{\circ} 18$ l'ombre de Zanco

$\mathrm{N}^{\circ} 19$ Le signe du cloporte

$\mathrm{N}^{\circ} 20$ le championnat de première division

$\mathrm{N}^{\circ} 21$ Le mauvais œil

$\mathrm{N}^{\circ} 22$ La coupe d'Europe

$\mathrm{N}^{\circ} 23$ les 7 vents de Wendigo

$\mathrm{N}^{\circ} 24$ le dévoreur des ténèbres

$\mathrm{N}^{\circ} 25$ Le messager du diable

$\mathrm{N}^{\circ} 26$ Poil de carotte

$\mathrm{N}^{\circ} 27$ Le défi

N²8 Les bananes flambées

$\mathrm{N}^{\circ} 29$ L'équipe de va-nu-pieds

$\mathrm{N}^{\circ} 30$ Les mascottes

$\mathrm{N}^{\circ} 31$ Question de coupe

N³2 Chantage publicitaire (Romano a formé une équipe de Lengros et les entraîne pour la coupe des jeunes qu'ils détiennent depuis trois ans, mais Horace Haslam, l'entraîneur de Dunsdeen, l'équipe adverse est prête à tout pour l'empêcher de gagner. Pour ce faire, il s'offre les services d'un hypnotiseur. Celui-ci hypnotise Romano afin qu'il perde ses moyens à chaque fois qu'il voit le maillot noir du goal...)

No33 Romano super star (Grafting, le fabricant de chaussures de sport a sauvé la vie de Romano suite à une machination et lui a ainsi extorqué 3 voeux. Le 1er sera de porter ses chaussures afin d'en faire la publicité. Romano, habitué à jouer pieds nus se soumet à sa promesse (bien encouragé par Morag), mais cela ne se fait pas sans difficulté...)

\section{$\mathrm{N}^{\circ} 34$ La vengeance}

$N^{\circ} 35$ Le proscrit

\section{$N^{\circ} 36$ Pour la coupe}

$\mathrm{N}^{\circ} 37$ Les loulous de la décharge (Alors que Romano anime un stand dans une fête de Lengros, un gardien de but parvient à arrêter un de ses tirs et obtient en échange sa participation à un match de bienfaisance. Malheureusement, il y rencontre Gary Logan, un excellent joueur, mais doté un ego surdimensionné. La rivalité s'installe aussitôt avec Romano et ce dernier fera tout pour épater son adversaire, y compris faire construire une maison gigantesque quitte à négliger son équipe ...) 


\section{$N^{\circ} 38$ Le talisman}

$\mathrm{N}^{\circ} 39$ Le signe du corbeau

$\mathrm{N}^{\circ} 40 \mathrm{La}$ tache noire

$\mathrm{N}^{\circ} 41$ Le faucon des Everglades

$\mathrm{N}^{\circ} 42$ Le troisième oril

$\mathrm{N}^{\circ} 43$ Pouce en bas

$\mathrm{N}^{\circ} 44$ Le roi du pistolet

$\mathrm{N}^{\circ} 45$ Dribble en solo

$\mathrm{N}^{\circ} 46$ Le match de la vérité

\section{Annexe}

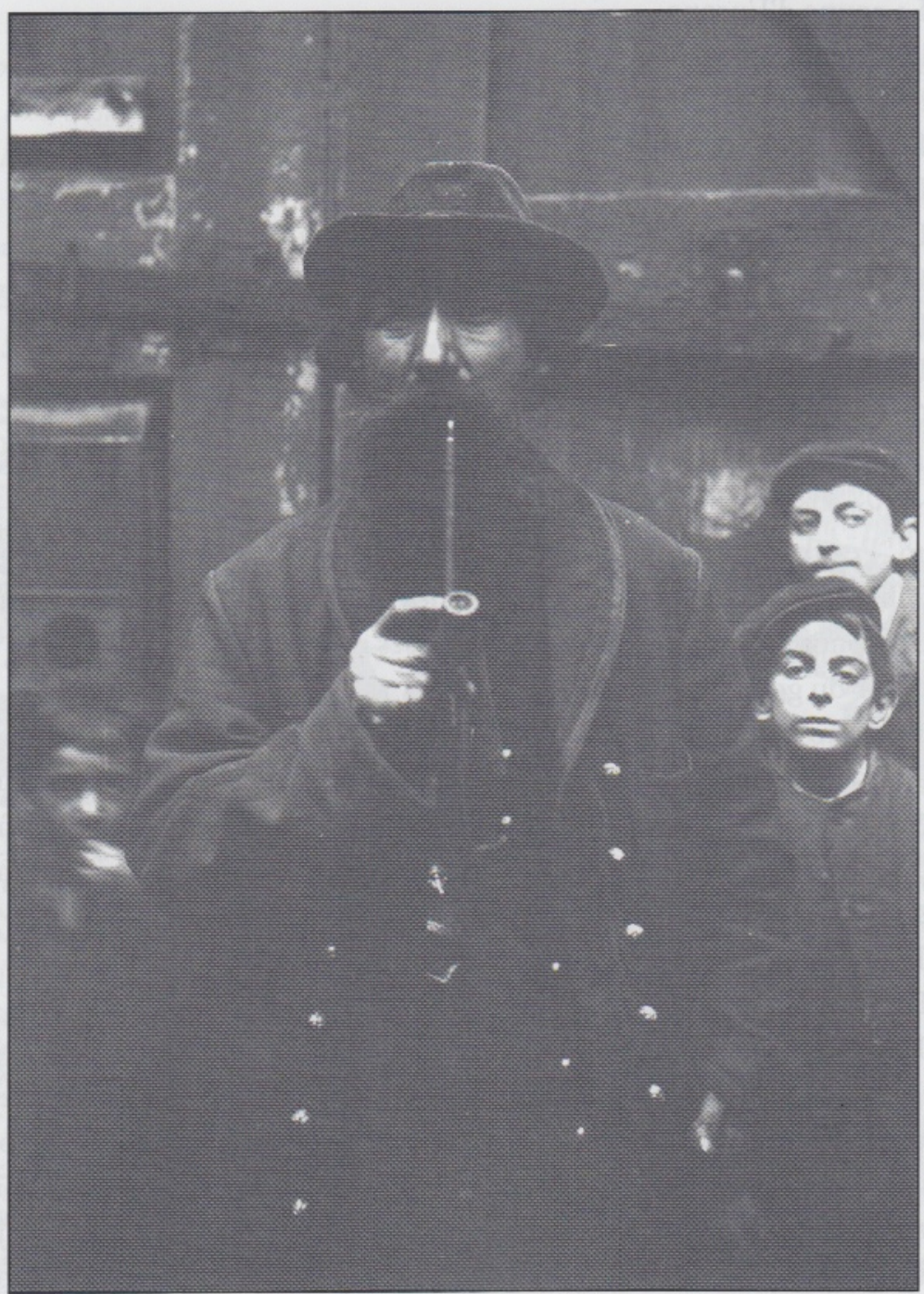

Photographic intitulée « Zanco » extraite des archives de la Gypsy Lore Society, 1914 (C) Estate of Fred Shaw. By courtesy of the University of Liverpool Library ; Fred Shaw Gypsy Photos : SMGC 1 / 2 Shaw P.89 


\section{Notes}

1. http://www.pimpf.org/mjm/trophee.htm http://www.bedetheque.com/serie-9849-BD-Trophee.html http://bdmonjournal.free.fr

2. Id.

3. Comme dans nombre de bandes dessinées la graphie des textes est en capitale, on ne peut donc rien savoir, ni déduire, de celle-ci; si par exemple il y a une différence entre un «manouche » nom commun équivalent à un "gitan » ou un "Manouche » distingué d'un " Gitan » comme il peut se faire en typographie française ordinaire, mais qui posent toujours problème aux secrétaires de rédaction. Voir à ce sujet les articles et textes de Patrick Williams.

4. Cf. Bordigoni Marc, Binisti Nathalie, 2002,»Mon gadjo, le gitan ...», emprunt lexical et reconfiguration catégorielle, in: Colloque «Variation, catégorisation et pratiques discursives", SYLED et Centre de recherche sur le français contemporain, Paris III Sorbonne Nouvelle, Paris, Langage et Société, consultable en ligne : https://halshs.archives-ouvertes.fr/halshs-00004000

5. Le patronyme Zanko est celui de familles roms présentes en France et ailleurs, bien sûr. Certaines sont passées en Angleterre dès les années 1910, ont séjourné aux alentours de Londres. Le scénariste aura pu lire dans la presse de l'époque ou plus tard, ce nom et l'utiliser, ou plus simplement a-t-il rencontrer un ou des Zanco; l'allure de ces Roms, sûrement bien différente des Gypsies, a pu lu apparaitre comme celle « des gitans les plus primitifs d'Europe ».

On peut aussi rappeler que Denis Zanko est un footballeur et entraineur français (1984-1995); il ne faisait pas partie de la tribu des Vengali et ne jouait pas pieds nus. (sources Sacha Zanko et Wikipédia).

\section{Source Adèle Sutre.}

7. Williams Patrick, 1997, «L'écriture entre l'oral et l'écrit : six scènes de la vie tsigane en France», in Fabre D. (Ed.), Par écrit : Etthnologie des écritures quotidiennes, Paris, Éditions de la Maison des sciences de l'homme-78, p. 59.

8. Sur le site de l'IDEMEC à la rubrique Documentaires - Atelier « Présences tsiganes » (http://www.idemec.cnrs.fr/spip.php?rubrique 70) est disponible un long entretien avec le footballeur gitan Jacques «Pancho » Abardonado réalisé par Roland Cottet en 2004. Il s'agit du rush intégral de cet entretien en famille dont une courte séquence a été utilisée par Cottet pour le film "Gitans, Tziganes, Gens du Voyage, le droit de vivre ... », diffusé sur France 5 en 2004. 
